\title{
Problèmes posés par la convection mixte du sodium dans les réacteurs rapides Modélisations physique et numérique
}

\author{
Mixed sodium convection problems in fast reactors \\ Laboratory and mathematical model simulation
}

\author{
Dominique Grand, Roland Vidil \\ CEA-CEN Grenoble
}

Dominique Taillifet, Jean-Pierre Benque

E.D.F.-LNH Chatou

\section{Notations}

Bo nombre de Boussinesq éq. 9

Fr nombre de Froude éq. 7

$g$ accélération de la pensanteur

Gr nombre de Grashof

$L \quad$ longueur caractéristique

$\vec{n} \quad$ vecteur unitaire normal à la frontière du domaine

$p^{*}$ pression réduite

Pe nombre de Péclet éq. 6

Pr nombre de Prandtl éq. 8

Re nombre de Reynolds éq. 8

$t$ temps

$T$ température

$\Delta T$ écart de température caractéristique

$U$ vitesse caractéristique

$U_{0}$ vitesse en entrée du domaine

$\vec{V}$ vitesse locale

$z$ cote

$\alpha$ diffusivité thermique

$\beta$ coefficient de dilatation cubique

$\lambda$ conductivité moléculaire

$\nu \quad$ viscosité cinématique

$\rho$ masse volumique

$\tau$ temps caractéristique

$\phi$ flux thermique

Nota :

$\hat{G}$ : rapport entre les valeurs prises dans un modèle et dans la réalité par une grandeur physique $G$.

$\mathrm{m}^{2} / \mathrm{s}$

$\mathrm{K}^{-1}$

$\mathrm{W} / \mathrm{m} \times \mathrm{K}$ $\mathrm{m}^{2} / \mathrm{s}$

$\mathrm{kg} / \mathrm{m}^{3}$

$\mathrm{s}$

$\mathrm{W} / \mathrm{m}^{2}$

\section{Introduction}

Le développement des réacteurs rapides refroidis par le sodium et conçus suivant le type intégré donne une grande importance à la thermohydraulique des grands volumes de sodium.

Le but de ces études est d'estimer les distributions de température auxquelles sont soumises les structures (cuves-composants) au cours des modes de fonctionnement rencontrés dans le réacteur.

L'examen des cas concrets pour lesquels cette connaissance des champs de température est nécessaire montre qu'une première distinction peut être faite suivant que le champ des vitesses est couplé ou non au champ des températures.

Ceci est mis en évidence par une réflexion sur les nombres adimensionnels qui tient compte des caractères particuliers de la thermohydraulique dans les métaux liquides.

Les moyens mis en œuvre pour résoudre ces problèmes au Service des Transferts Thermiques (CEACENG) et au Laboratoire National d'Hydraulique (EDF) sont présentés dans cette optique.
Analyse dimensionnelle

\section{Les équations}

La thermohydraulique d'un fluide incompressible dans un domaine $\delta$ quelconque est régie par :

- des équations de conservation

masse :

$$
\operatorname{div} \vec{V}=0
$$


quantité de mouvement :

$$
\frac{\overrightarrow{d V}}{d t}=-\overrightarrow{\operatorname{grad}} p^{*}+\frac{v}{U L} \Delta \vec{V}+\frac{g \beta \Delta T L}{U^{2}} T
$$

chaleur :

$$
\frac{d T}{d t}=\frac{\alpha}{U L} \Delta T
$$

Parmi les variations des propriétés physiques du fluide avec la température, on n'a retenu que celle de la masse volumique, et seulement dans la force ${ }^{*}$ de volume : c'est l'hypothèse classique de Boussinesq.

Sans restreindre la généralité, on a rapporté les variables à des grandeurs caractéristiques : une longueur $L$, une vitesse $U$, et un écart de température $\Delta T$.

On a posé :

$$
p^{*}=\frac{p+\rho g z}{\rho U^{2}}
$$

- des conditions aux limites, qui peuvent être de la forme :

vitesse imposée :

$$
\vec{V} \cdot \vec{n}=\frac{U_{0}}{U}
$$

flux thermique imposé :

$$
\vec{n} \cdot \overrightarrow{\operatorname{grad}} T=\frac{\phi L}{\lambda \Delta T}
$$

\section{Grandeurs caractéristiques}

Le choix des grandeurs caractéristiques doit être fait de façon à ce qu'apparaissent des nombres adimensionnels pertinents. La géométrie que l'on étudie et le problème thermique posé avec ses conditions aux limites conduisent en général directement au choix de $L$ et $\Delta T$. Par contre, on ne connaît pas toujours a priori la vitesse qui caractériserait au mieux l'écoulement. On peut faire deux choix extrêmes:

- $U=U_{0}$. Ce choix correspond à une situation où la convection forcée régit l'écoulement. Les groupements adimensionnels apparaissent uniquement dans les équations de conservation. Ce sont :

- dans l'équation (3):

$$
\frac{\alpha}{U_{0} L}=P e^{-1}
$$

- dans l'équation (2):

$$
\frac{g \beta \Delta T L}{U_{0}^{2}}=F_{r}^{-1}
$$

- dans l'équation (2):

$$
\frac{\nu}{U_{0} L}=\frac{\nu}{\alpha} P e^{-1}=\operatorname{Pr} P e^{-1}=R e^{-1}
$$

Le nombre de Péclet, Pe, compare les puissances transmises par convection et par conduction thermique.

L'inverse du nombre de Froude, $\mathrm{Fr}^{-1}$, qui apparaît dans l'équation de la quantité de mouvement, caractérise l'importance relative des effets de densité (poussée d'Archimède) et des effets d'inertie. C'est par lui seul que la thermique peut influencer l'écoulement (dans 1'hypothèse de Boussinesq).

- Contrairement à un usage établi, le nombre de Reynolds est exprimé sous la forme du groupement $\mathrm{Pr}^{-1} \mathrm{Pe}$. Cette présentation a l'avantage de faire apparaître les conséquences de la faible valeur du nombre de Prandtl du sodium $\left(\operatorname{Pr} \simeq 5 \times 10^{-3}\right)$ :

- Les tensions visqueuses, de l'ordre de $\operatorname{Pr} P e^{-1}$, sont plus faibles qu'en fluide non métallique, toutes choses égales par ailleurs.

- Les flux de chaleur, dont l'ordre de grandeur est $P e^{-1}$ (équation (3)), deviennent grands dans le cas du sodium.

- $U=(g \beta \Delta T L)^{1 / 2}$. Ce choix correspond au cas où l'écoulement est amorcé ou régi par la convection naturelle, donc quand $U_{0}$ est nul ou faible.

Les groupements adimensionnels suivants apparaissent alors :

- dans l'équation (3):

$$
\frac{\alpha}{U L}=\frac{\alpha}{\left(g \beta \Delta T L^{3}\right)^{1 / 2}}=\left(G r P r^{2}\right)^{-1 / 2}=B O^{-1 / 2}
$$

- dans l'équation (2):

$$
\frac{\nu}{U L}=\frac{\nu}{\left(g \beta \Delta T L^{3}\right)^{1 / 2}}=\operatorname{Pr}\left(G r \operatorname{Pr}^{2}\right)^{-1 / 2}=\operatorname{Pr} B o^{-1 / 2}
$$

- dans l'équation (2):

$$
\frac{g \beta \Delta T L}{U^{2}}=1
$$

C'est ce qui a guidé le choix de $U$.

- dans la condition à la limite (4):

$$
\frac{U_{0}}{U}=\frac{U_{0}}{(g \beta \Delta T L)^{1 / 2}}=F r^{1 / 2}
$$

Le nombre de Boussinesq (terminologie proposée par Le Fèvre (1976)) Bo $=G r P^{2}$ compare les puis sances transmises par convection et par conduction thermique.

- La présence du nombre de Froude dans la condition à la limite montre sa signification comme rapport de la vitesse en entrée du domaine à celle de la convection naturelle.

- Le groupement $\operatorname{Pr} B o^{1 / 2}$ régit les tensions visqueuses. Celles-ci deviennent négligeables, dans la limite où $\operatorname{Pr}$ devient très petit.

\section{Mise en évidence dans le cas des couches limites}

Il a été vérifié par ailleurs, Grand et Vernier (1979), que dans le cas où le nombre de Prandtl est petit, il a 
une influence évanescente sur le champ de température quand on retient comme paramètre adimensionnel : - Bo en convection naturelle,

- Pe en convection forcée.

Ceci a pu être vérifié dans le cas des couches limites de paroi pour lesquelles on dispose de résultats en métaux liquides.

\section{Ordres de grandeur dans les réacteurs}

Nous situons maintenant les problèmes concrets relatifs aux réacteurs rapides, à l'aide des nombres adimensionnels présentés plus haut. Le tableau suivant les présente. La vitesse de référence $U_{0}$ sur laquelle sont construits $P e$ et $F r$ est ici la vitesse imposée à l'entrée du domaine concerné.

\begin{tabular}{|c|c|c|c|c|c|c|c|}
\hline $\begin{array}{l}N^{\circ} \\
\text { du } \\
\text { cas }\end{array}$ & Description du problème & $\begin{array}{l}U_{0} \\
\quad(\mathrm{~m} / \mathrm{s})\end{array}$ & $L$ & $\begin{array}{l}\Delta T \\
\quad(\mathrm{~K})\end{array}$ & $P_{e}$ & $\mathrm{Fr}$ & Bo \\
\hline 1 & $\begin{array}{l}\text { Echangeur Intermédiaire } \\
\text { circuit primaire }\end{array}$ & 1,8 & 0,02 & 50 & $5,6 \times 10^{2}$ & 1200 & 270 \\
\hline 2 & $\begin{array}{l}\text { Super Phénix - Collecteur } \\
\text { froid - régime nominal }\end{array}$ & 1,8 & 4,5 & 10 & $1,3 \times 10^{5}$ & 26 & $6,1 \times 10^{8}$ \\
\hline 3 & $\begin{array}{l}\text { Super Phénix - Collecteur } \\
\text { chaud - Arrêt d'urgence au } \\
\text { régime nominal }\end{array}$ & 3 & 5 & 115 & $2,3 \times 10^{5}$ & 5,7 & $9,6 \times 10^{9}$ \\
\hline 4 & $\begin{array}{l}\text { Super Phénix - Collecteur } \\
\text { chaud - Arrêt d'urgence à } \\
\text { mi-débit }\end{array}$ & 1,5 & 5 & 100 & $1,2 \times 10^{5}$ & 1,6 & $8,4 \times 10^{9}$ \\
\hline 5 & $\begin{array}{l}\text { Phénix - Collecteur froid - } \\
\text { Régime nominal }\end{array}$ & 0,6 & 3 & 80 & $2,8 \times 10^{4}$ & 0,55 & $1,4 \times 10^{9}$ \\
\hline 6 & Super Phénix. Corps mort. & 0,2 & 1 & 150 & $3,1 \times 10^{3}$ & 0,10 & $1.0 \times 10^{8}$ \\
\hline 7 & Super Phénix. DCNEP & 0,01 & 5 & 35 & $7,8 \times 10^{2}$ & $2 \times 10^{-4}$ & $2,9 \times 10^{9}$ \\
\hline 8 & $\begin{array}{l}\text { Super Phénix. } \\
\text { Espace inter-redan. }\end{array}$ & - & 2 & 35 & - & - & $1,9 \times 10^{8}$ \\
\hline
\end{tabular}

Ce tableau montre l'extrême variété des problèmes rencontrés, en particulier du point de vue du nombre de Froude. Notons par exemple que le collecteur froid de Phénix se comporte très différemment (couplage intense) de celui de Super Phénix où les écoulements sont pratiquement indépendants de la thermique. Cette différence tient essentiellement à l'accroissement de la vitesse à la sortie de l'échangeur.

On remarque également que dans certains cas, comme celui de l'écoulement primaire dans l'échangeur intermédiaire, le nombre de Froude très grand est à associer à des phénomènes complètement découplés.

Il apparaît donc que l'on peut classer les problèmes d'écoulement de sodium dans les réacteurs rapides, et les moyens mis en œuvre pour les résoudre, selon trois catégories, selon que les problèmes sont découplés (grand nombre de Froude) ou couplés (petit nombre de Froude). Dans ce dernier cas, on peut faire une distinction supplémentaire selon que la conduction influence ou non le champ thermique.

Les difficultés ne sont pas de même nature dans ces classes de problèmes. Dans le cas des problèmes découplés les écoulements sont souvent tridimensionnels et très sensibles à la géométrie, la formation de sillages et de décollements, les répartitions de vitesses et les mélanges prenant de l'importance. Dans le cas de faibles vitesses et de couplage intense, le problème est souvent plus bidimensionnel du fait de la particularisation de la direction verticale, et moins sensible à la géométrie. Cependant, la prise en compte des effets de la conduction est souvent difficile.

$\mathrm{Si}$ cette classification est une approche utile, elle ne fournit pas toujours un partage net entre les problèmes rencontrés. Ainsi, la distinction suivant que la conduction a une influence ou non, ne repose pas sur un critère quantitatif précis et doit faire l'objet d'un examen cas par cas.

\section{Classification des problèmes}

\section{Problèmes découplés}

\section{PRESENTATION}

Le nombre de Froude est suffisamment grand pour que l'écoulement puisse être étudié indépendamment du champ de températures. Au régime nominal, les zones des cuves quasi isothermes (collecteur chaud et froid de Super-Phénix), et les échangeurs de chaleur présentent cette particularité, ainsi que les écoulements dans le cœur. 


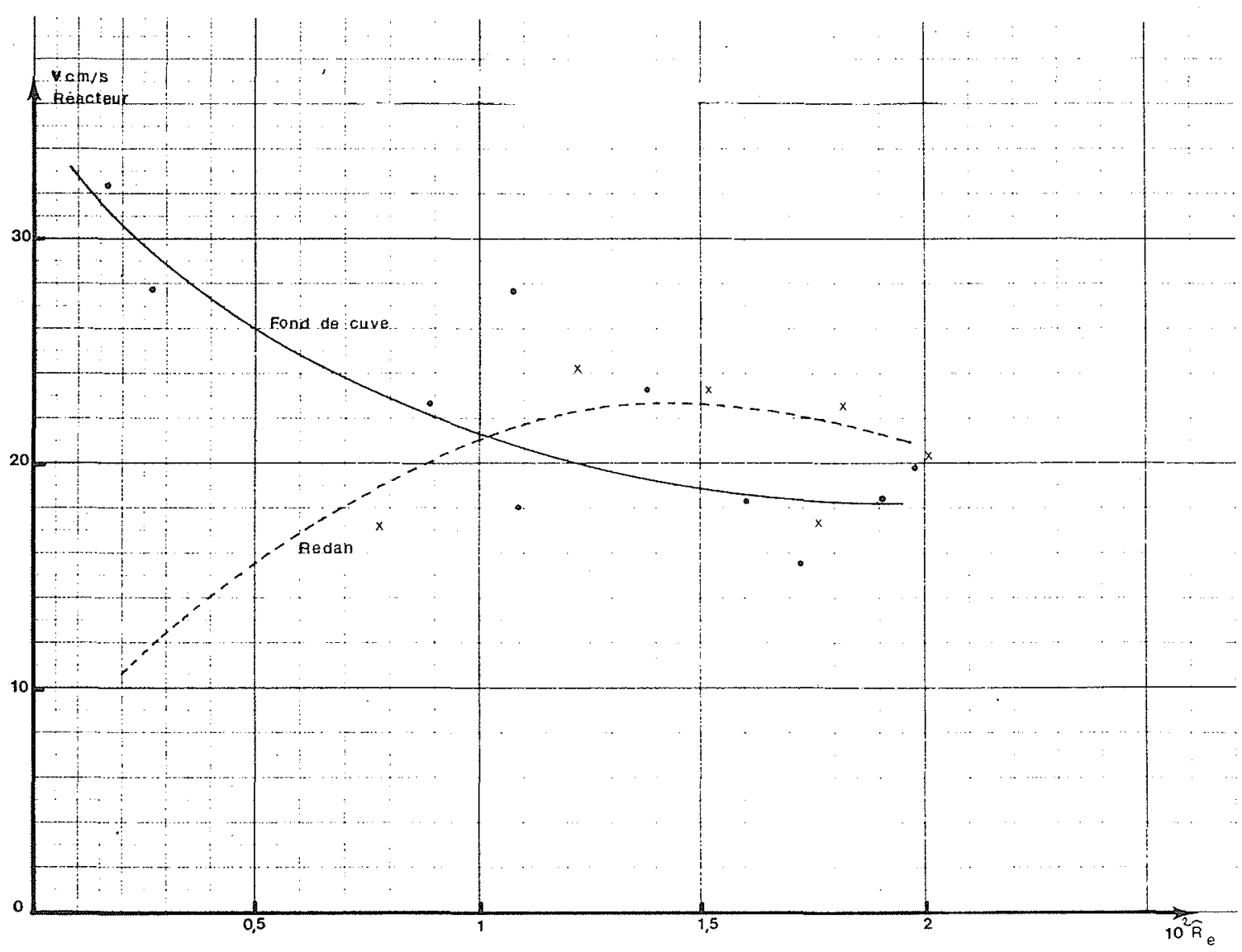

Figure 1. - Collecteur froid - échelle 1/10. Effet de $R_{e}$ sur l'écoulement isotherme.

Le nombre de Reynolds $(\mathrm{Pe} / \mathrm{Pr})$ est généralement grand dans ces cas. On peut souvent distinguer des plages de valeur de ce nombre caractérisant des régimes d'écoulement mais à l'intérieur desquelles son influence n'est pas déterminante. C'est le cas des écoulements engendrés par un jet et de ceux où peuvent apparaître des décollements.

\section{METHODES D'APPROCHE}

\section{A) Etude de l'écoulement}

Les écoulements sont étudiés par deux types de moyens :

\section{A-a) Modèles physiques}

Les modèles physiques (Fig. 1) permettent de représenter fidèlement les géométries tridimensionnelles.

On retient en général des modèles en eau, qui présentent des avantages de mise en œuvre et d'exploitation.

L'échelle de ces modèles est souvent voisine de $1 / 15$ ou $1 / 10$ pour un modele d'ensemble, mais peut être $1 / 2$ ou 1 pour un modèle de détail. Leurs parois en altuglass permettent des visualisations qui sont précieuses pour la compréhension des écoulements.
Les moyens performants dont nous disposons maintenant (capteurs de pression et vélocimètre à laser) permettent de connaître l'écoulement moyen et ses fluctuations (du moins en isotherme).

Il est rarement possible de vérifier

$$
\widehat{R} e=\frac{R e_{\text {(modèle) }}}{R e_{(\text {prototype) }}}=1
$$

Un exemple d'influence de la distorsion du nombre de Reynolds est donné figure 2. La vitesse est mesurée en un point près du redan et un point en fond de cuve d'un modèle au $1 / 10 \mathrm{du}$ collecteur froid d'un réacteur rapide. On voit que la vitesse ramenée en grandeur prototype est influencée par le débit du modèle en dessous $\operatorname{de} \hat{R} e=1 / 100$.

\section{A-b) Modèles numériques}

Les modèles numériques sont en plein développement et prennent d'ores et déjà une place importante dans ces études.

On résoud, à l'aide d'un ordinateur, les équations de Navier Stokes par des méthodes de différences finies mais sans approximation particulière. 


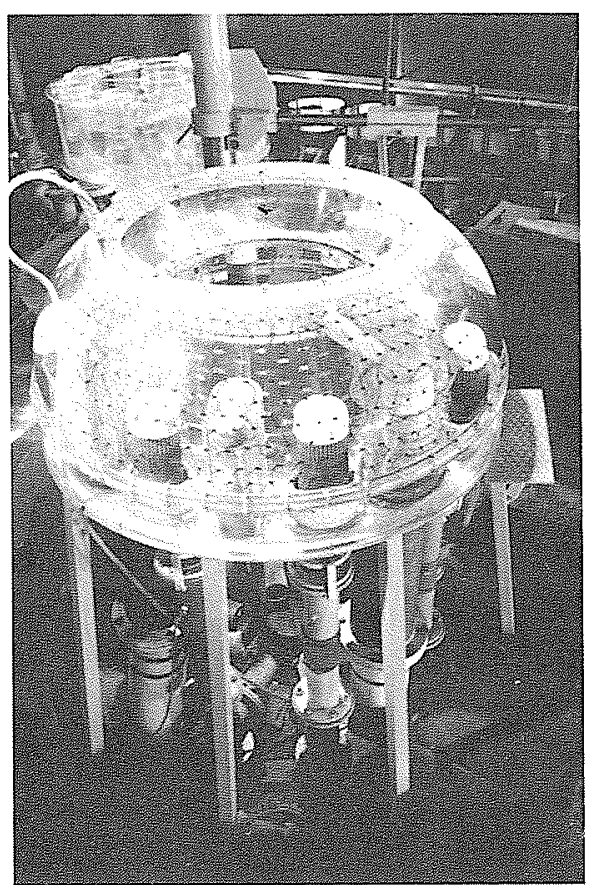

Figure 2 - Super Phénix. Modèle physique au 1/15.

\section{B) Etude de la thermique}

On utilise le champ des vitesses obtenu par les méthodes au $\S \mathrm{A}$ :

- pour estimer des valeurs de coefficients d'échanges quand il s'agit de déterminer des flux entre grandes zones pratiquement isothermes,

- pour calculer le champ de températures, compte tenu des conditions aux limites, souvent transitoires, des convections et éventuellement de coefficients d'échanges aux parois.

\section{Problèmes couplés sans influence de la conduction}

\section{PRESENTATION}

Il s'agit essentiellement ici des transitoires rapides. En effet, pour un problème à étudier pendant quelques minutes, les flux convectés par le premier terme de l'équation (3) régissent l'évolution des températures, le second membre étant d'un ordre de grandeur beaucoup plus petit. Les conditions initiales permanentes étant données, la conduction n'a donc pas le temps d'influencer l'évolution transitoire.

\section{METHODES D'APPROCHE}

\section{A) Modeles physiques}

Ils peuvent être de deux types:

- modèle en eau douce - eau salée

Les modèles présentés plus haut peuvent être utilisés pour ces problèmes thermohydrauliques. Les écarts de densité dus au champ thermique peuvent être simulés par des écarts de concentration saline, en similitude de Froude. Cette méthode permet de garder pendant les essais thermohydrauliques un nombre de Reynolds élevé, ce qui est particulièrement important pour une bonne représentation des jets et des écoulements en général. Notons cependant que de tels modèles sont souvent, de ce point de vue, à la limite inférieure au-dessous de laquelle on ne pourrait descendre sans risque de mal représenter l'écoulement. Ainsi, dans les modèles au $1 / 15$ de Super Phénix, on a pendant les essais présentés en application $\hat{R} e=1 / 200$.

La transposition directe des concentrations mesurées permet de donner la température du sodium au-delà des couches limites. Il faut ensuite tenir compte de celles-ci pour obtenir les températures de peau ce qui, de toutes façons, ne conduit qu'à une correction de quelques degrés à comparer à l'amplitude des transitoires considérés, souvent de l'ordre de $100^{\circ} \mathrm{C}$.

Cette méthode présente donc l'avantage de ne pas distordre de façon trop importante le nombre de Reynolds.

Il faut toutefois bien s'assurer que la conduction n'a pas d'influence dans le réel, le champ de températures obtenu étant imprécis dans le cas contraire.

\section{- modèle en eau douce froide - eau chaude}

Dans ces problèmes, les modèles en eau douce/eau chaude peuvent également être utilisés en similitude de Froude avec $\widehat{R} e=1 / 200$, et un début de représentation des effets de conduction $(\hat{P e}=5)$. Ceci conduit à des modèles dont l'échelle est assez grande $(\hat{L} \sim 1 / 10)$.

Dans le cas où l'on veut améliorer la représentation des effets de conduction, en respectant la similitude de Péclet, on utilise des modèles à échelle plus petite $(\widehat{L} \sim 1 / 20)$. On rencontre alors l'inconvénient d'une distorsion supérieure du nombre de Reynolds ( $\widehat{R e}=$ $\widehat{P r}=1 / 1000)$, qui demande une vigilance accrue quant au respect des écoulements.

On peut alors étudier les transitoires pour des temps plus longs où la conduction joue :

- pour détruire une éventuelle stratification,

- dans le couplage de deux collecteurs par l'intermédiaire des parois.

Ces modèles peuvent également être envisagés dans le cas de conditions initiales franchement non isothermes.

\section{B) Modèles numériques}

Dans le cas des régimes transitoires, les effets tridimensionnels et l'importance de la prise en compte exacte des volumes de sodium concernés interdisent l'emploi d'une modélisation bidimensionnelle. On peut dans ce cas utiliser le modèle tridimensionnel, comme il sera précisé au niveau des applications. Cette approche permet d'obtenir un chargement thermique des structures pratiquement continu (à la discrétisation du maillage près), alors que les essais ne peuvent donner les températures qu'en un nombre limité de points.

\section{Problèmes couplés avec influence de la conduction}

\section{PRESENTATION}

Ici, le nombre de Péclet et le nombre de Froude sont petits. Ce sont les cas permanents à faible vitesse tels 
que la zone du corps mort, l'espace inter-redan et les écoulements dans les divers doigts de gants ou cheminées. Cela peut également être un régime transitoire où la conduction peut avoir une influence :

- dans l'établissement des conditions initiales si elles sont franchement non isothermes,

- pour détruire une éventuelle stratification,

- dans le cas de couplage des deux collecteurs par l'intermédiaire des parois, (ces deux dernières remarques concernent l'évolution du fluide au-delà de quelques minutes lors d'un transitoire).

\section{MODELES PHYSIQUES}

Dans ce cas, le respect des deux nombres $P e$ et $F r$ est indispensable (cela revient à respecter $F r$ et $B o$ ).

Une méthode très satisfaisante consisterait à utiliser un métal liquide. Avec du mercure, l'échelle d'un tel modèle serait $1 / 5$.

Une autre méthode consisterait à utiliser de l'air sous pression qui permet également de satisfaire aux conditions de similitude.

Ces deux méthodes, éventuellement applicables pour des essais de confirmation, ne sont pas utilisées dans les études actuelles, d'importantes difficultés technologiques restant à résoudre.

Une méthode approchée plus facile à mettre en œuvre utilise des fluides non métalliques. L'échelle du modèle est alors comprise entre $1 / 30$ et $1 / 10$ avec de l'eau à la température ambiante. L'utilisation d'un tel fluide conduit à une importante distorsion du rapport $\mathrm{Pe} / \mathrm{Pr}$ (nombre de Reynolds) égale à $\hat{P} r$ puisque $\widehat{P} e=1$. C'est pourquoi il est souvent nécessaire de compléter l'étude par des essais paramétriques réalisés en augmentant le nombre de Péclet du modèle.

\section{MODELES NUMERIQUES}

La modélisation numérique des équations instationnaires se heurte à la difficulté suivante : le pas de temps est limité par la convection au niveau de la maille et généralement petit alors que le temps d'établissement du champ de température est imposé par la conduction.

De tels calculs sont cependant entrepris dans certains cas, comme il sera dit au niveau des applications.

\section{Exemples d'études de problèmes découplés}

\section{Collecteur chaud de Super Phénix au régime nominal}

Un modèle physique au $1 / 15$ a été mis en œuvre dans ce cas.

Parallèlement, le modèle numérique tridimensionnel a été appliqué. Il représente, en coordonnées cylindriques, $1 / 8$ du collecteur chaud, le coeur ayant été recentré.

On trouve figure 3 le champ de vitesses dans deux plans verticaux (1-2). Le plan 1 représente l'écoulement entre une pompe et un échangeur. On peut constater que le jet issu du cœur est "pincé" par l'échan-
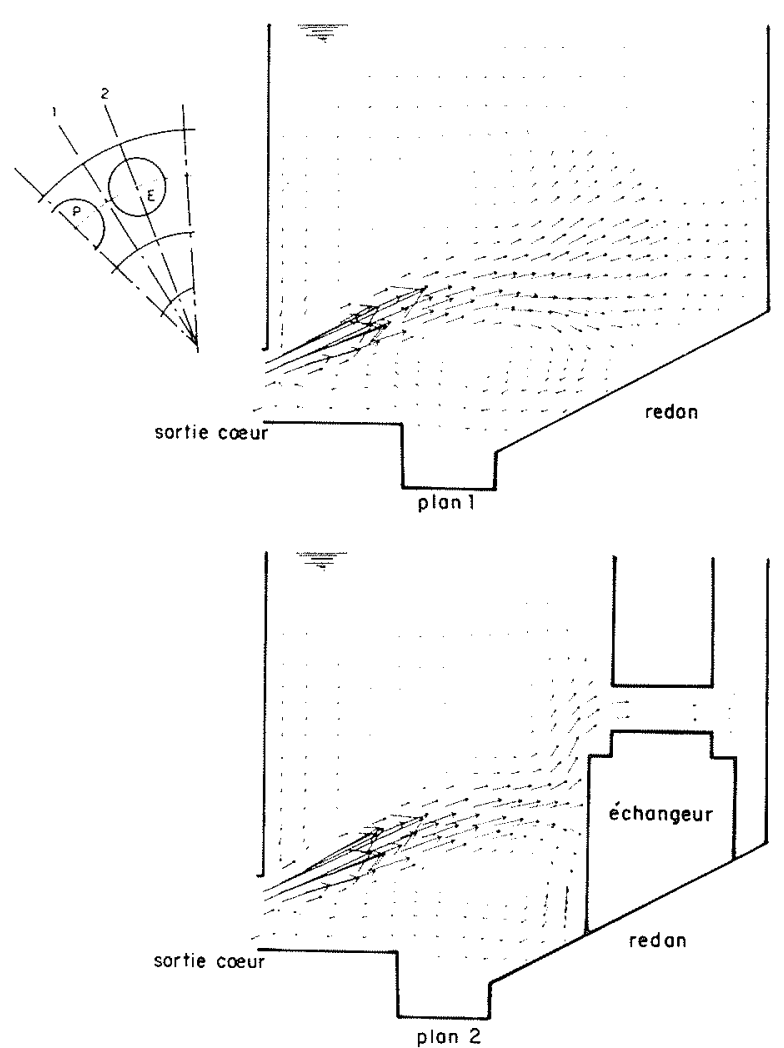

Figure 3 - Calcul tridimensionnel appliqué au collecteur chaud de Super-Phénix. Champ des vitesses au régime nominal.

geur et la pompe. Ce comportement a été observé également dans les expériences sur modèle réduit.

Dans le plan 2, le jet du.cœur vient frapper l'échangeur de chaleur et la recirculation vers le haut est nettement différente de ce que l'on observe dans le plan 1 où le jet plus libre vient frapper le baffle vertical de la cuve principale. Les mesures partielles dont on dispose ne permettent pour l'instant de ne s'assurer que d'un accord qualitatif avec les visualisations obtenues sur le modèle physique.

Dans ce cas, le collecteur est pratiquement isotherme et l'étude de la thermique se résume au couplage avec l'espace inter-redan, ce qui se fait à l'aide de coefficients d'échange trouvés dans la littérature.

\section{Echangeur intermédiaire}

Deux calculs axisymétriques sont utilisés dans ce cas, l'un concernant l'hydrodynamique, l'autre la thermique.

Du point de vue hydrodynamique, le faisceau de tube est schématisé par une porosité et des frottements homogènes et non isotropes. On peut alors écrire des équations déduites de celles de Navier Stokes, compte tenu de ces paramètres. Ces équations sont résolues par une méthode aux différences finies.

Les résultats ont pu être vérifiés à l'aide d'un modèle physique par comparaison des champs de vitesses mesuré et calculé (fig. 4). On constate une bonne concordance.

Du point de vue thermique, le calcul des circuits primaires et secondaires est effectué simultanément, le 


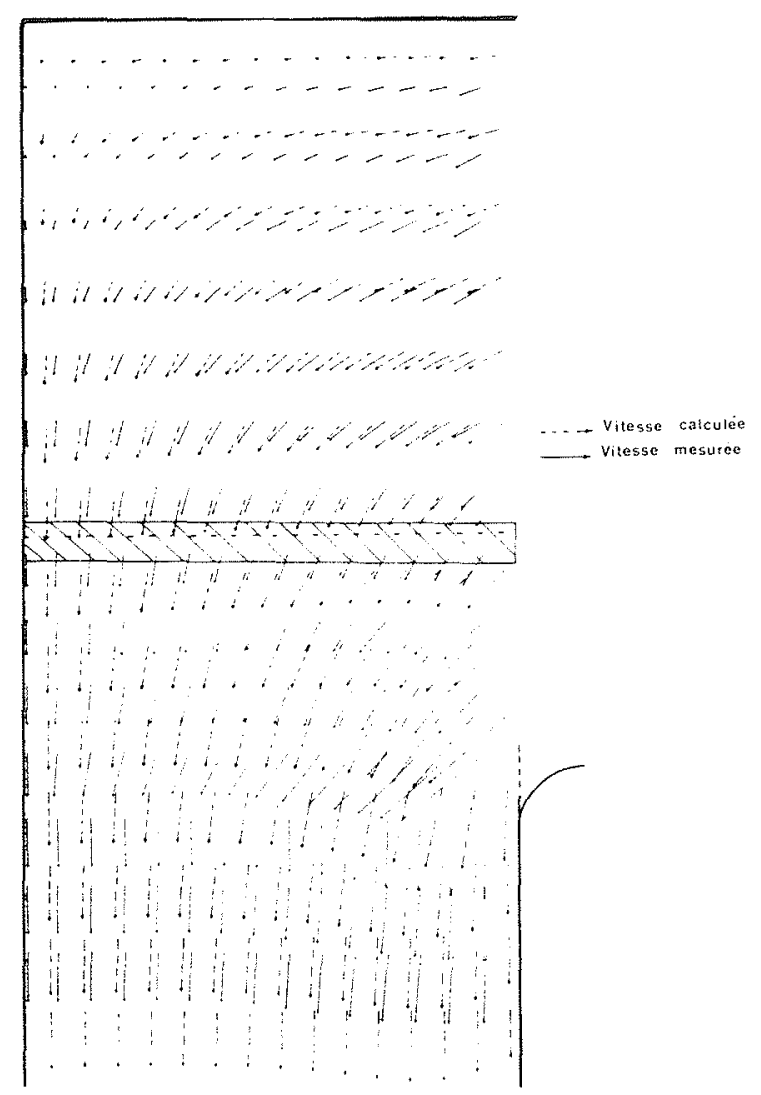

Figure 4 - Etalonnage du modèle Faituvit : Comparaison des champs de vitesse mesuré et calculé dans la zone d'entrée.

couplage étant obtenu à partir de coefficients d'échanges connus par ailleurs.

Cette méthode a permis d'analyser et de comprendre les répartitions de températures mesurées sur l'échangeur de Phénix et joue actuellement un rôle important dans la connaissance des répartitions de températures permanentes et transitoires à prendre en compte dans le dimensionnement du projet Super Phénix.

Exemple d'étude de problèmes couplés sans influence de la conduction : l'arrêt d'urgence dans Super Phénix

\section{Présentation du problème}

Le collecteur chaud de Super Phénix est, nous l'avons $\mathrm{vu}$, pratiquement isotherme au régime nominal. Lors d'un arrêt d'urgence, le sodium à la sortie du cœur devient brusquement froid, le débit étant maintenu constant. Il s'agit de déterminer comment ce front froid se répand dans la cuve, principalement en vue de connaitre les sollicitations thermiques des structures dans un tel cas.

\section{Utilisation des modèles physiques}

Le modèle au $1 / 15$ de Super Phénix a été utilisé selon la méthode en eau salée. Un exemple de résultat est indiqué figure 5, où l'on compare l'évolution du
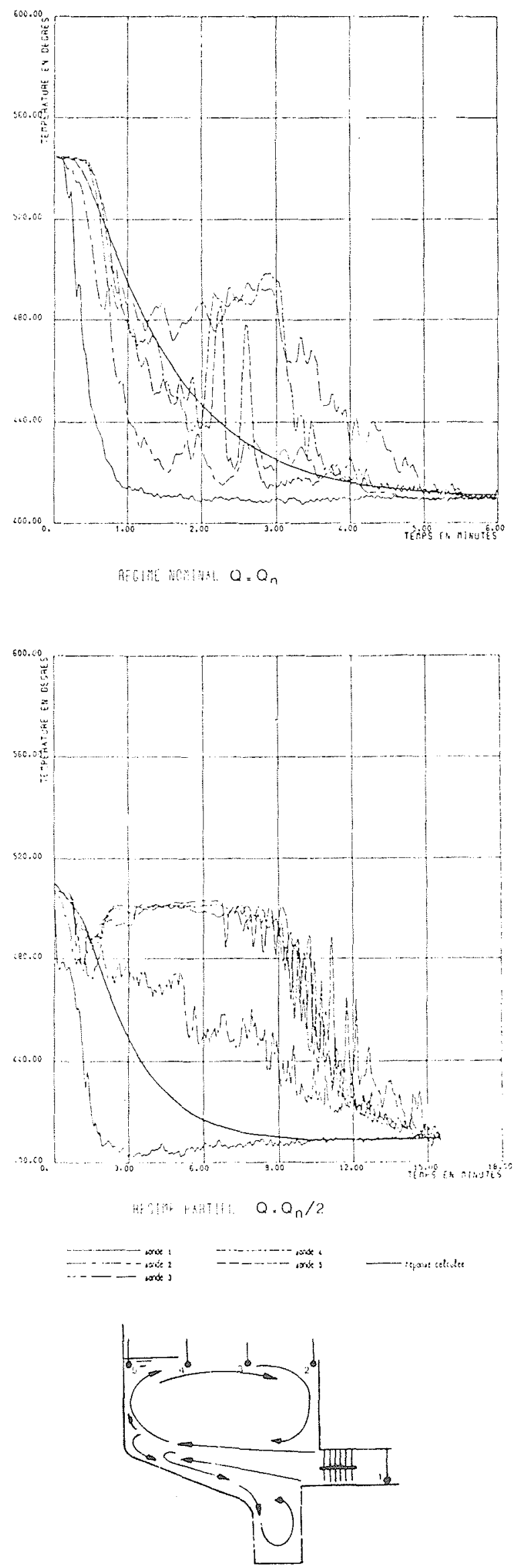

Figure 5 - Super-Phénix. Evolution des températures en arrêt d'urgence. 
collecteur pour deux nombres de Froude différents : le régime nominal et le régime partiel. On voit que dans ce dernier cas l'effet de densité est suffisamment important pour occasionner la formation d'une couche chaude.

Il est à noter qu'une telle couche chaude a été effectivement observée lors des essais de Phénix.

Un modèle, en eau chaude, à l'échelle $1 / 20$, a permis d'obtenir la mêtme distinction entre les deux régimes de fonctionnement : nominal et partiel, ce dernier conduisant ici encore à la formation d'une couche chaude.

\section{Utilisation du modèle numérique}

A partir du champ de vitesse tridimensionnel (cf. fig. 3), on a calculé un arrêt d'urgence au régime nominal, la température sortie cœur décroissant de $545^{\circ} \mathrm{C}$ à $395^{\circ} \mathrm{C}$ en 8 secondes.

$\mathrm{Si}$ on examine le champ de vitesses obtenu $20 \mathrm{se}$ condes après le début du choc thermique, on constate (Fig. 6) que le jet a été défléchi par les effets de gravité.

Sur la figure 7 , on a porté le champ thermique au même instant, dans les plans 1 et 2 . On constate que le jet a traversé le collecteur et est venu frapper les structures mécaniques.

La figure 8 présente une comparaison entre le résultat de ce calcul et celui de l'essai en sel. Il s'agit de l'évolution observée en deux points sur le redan. Il apparaît que les temps de transit sont en accord ainsi que la décroissance de température.
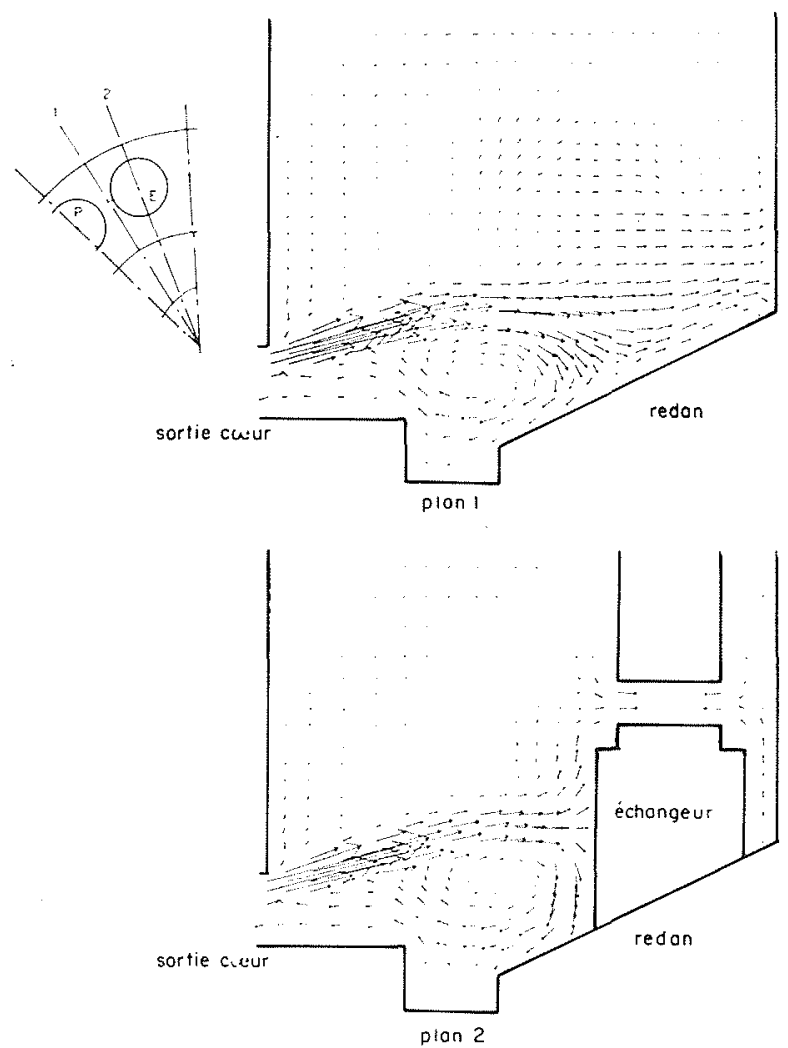

Figure 6 - Calcul tridimensionnel appliqué au collecteur chaud de Super-Phénix. Champ des vitesses lors d'un arrêt d'urgence $(t=20 \mathrm{~s})$.
Ces résultats encourageants doivent être confirmés avant une utilisation plus systématique du code pour traiter les divers transitoires rapides.
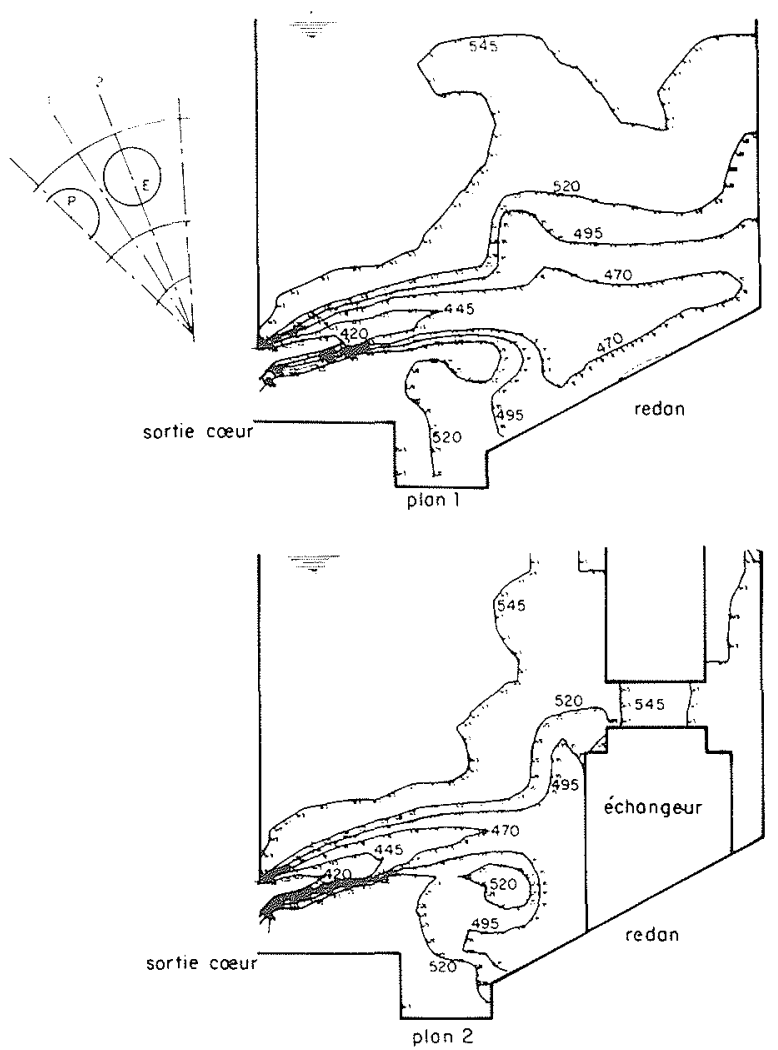

Figure 7 - Calcul tridimensionnel appliqué au collecteur chaud de Super-Phénix. Lignes isothermes lors d'un arrêt d'urgence $(t=20 \mathrm{~s})$.

Solinité sur le redon ou cours du choc froid
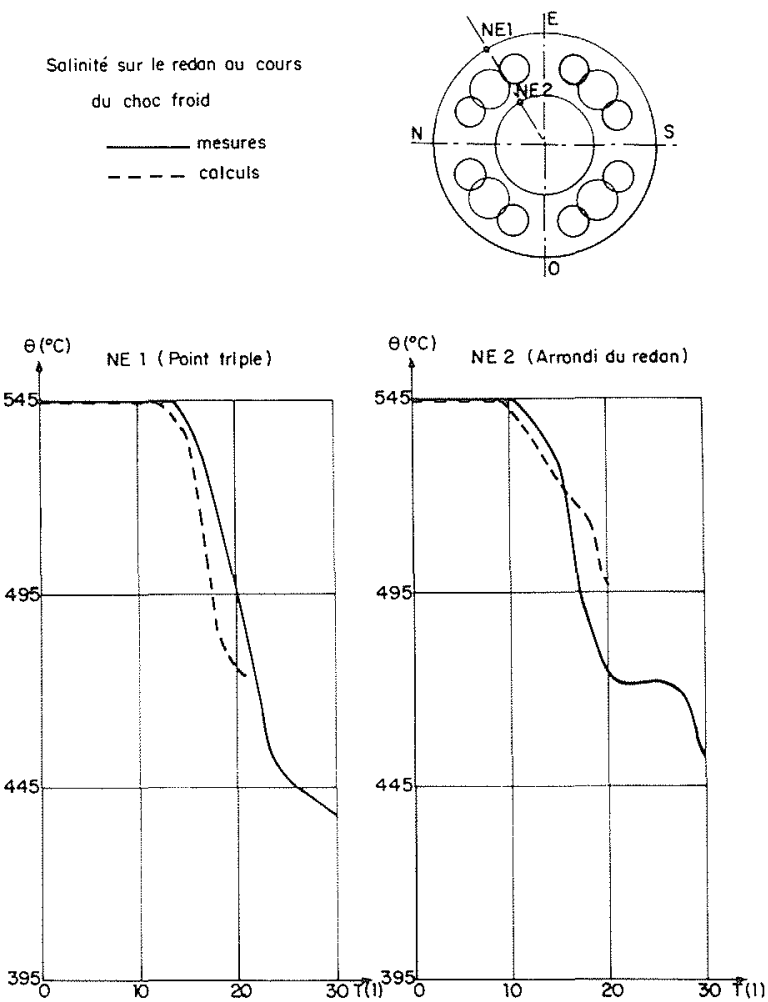

Figure 8 - Arrêt d'urgence. Comparaison calculs-mesures. 

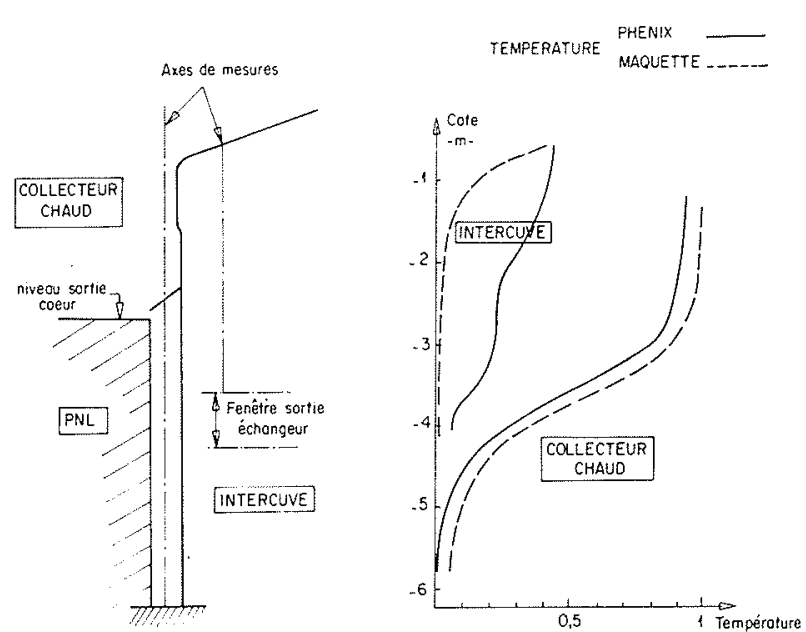

Figure 9 - Températures dans les collecteurs en régime permanent. Comparaison maquette-réacteur.

\section{Exemples d'études de problèmes couplés avec} influence de la conduction

\section{Champ thermique dans Phénix au régime nominal}

Un modèle physique en eau chaude a été utilisé dans ce cas, l'existence de relevés de températures dans le réacteur permettant d'apprécier la qualité de ses enseignements. Le modèle utilisé représente un secteur (1/6) du réacteur Phénix à l'échelle 1/33. La paroi en altuglas simulant la cuve interne est dimensionnée en respectant le rapport des résistances thermiques fluide et paroi.

Dans la figure 9 les résultats du modèle sont comparés aux mesures enregistrées sur Phénix au fonctionnement nominal à l'aide de 2 perches instrumentées installées, l'une dans le collecteur chaud entre la cuve interne et les Protections Neutroniques Latérales (PNL), l'autre dans l'intercuve.

\section{DISTRIBUTION DE TEMPERATURE DANS LA ZONE DES PNL}

La région comprise entre les PNL et la cuve interne forme une cavité très élancée. Le champ thermique y résulte :

- des recirculations chaudes induites en partie supérieure par le débit sortant du cœur,

- du refroidissement créé par les échangeurs à travers la paroi de la cuve interne.

Les distributions observées sur maquette concordent avec le relevé de Phénix.

\section{DISTRIBUTION DE TEMPERATURES DANS L'IN- TERCUVE.}

Dans le réacteur, on observe partant de la fenêtre de sortie des échangeurs :

- un gradient de température dans la couche de mélange du débit issu de l'échangeur,

- une température uniforme dans la recirculation,

- une stratification stable dans la partie supérieure.

Dans le modèle, la répartition entre régions turbulentes (couche de mélange et recirculation) et stratifiées est qualitativement retrouvée. Des différences apparaissent et elles sont dues à une répartition différente des résistances thermiques dans la couche de mélange et dans la stratification.

\section{Arrêt d'urgence dans super Phénix.}

Nous avons constaté précédemment un accord qualitatif des différentes approches quant à la détection de l'apparition d'une couche chaude dans la partie supérieure du collecteur chaud. Cette apparition était liée au nombre de Froude seul. Cependant son évolution ultérieure peut être conditionnée par les effets de conduction et un modèle thermique- est actuellement le moyen le plus adapté à son étude. La figure 10 montre

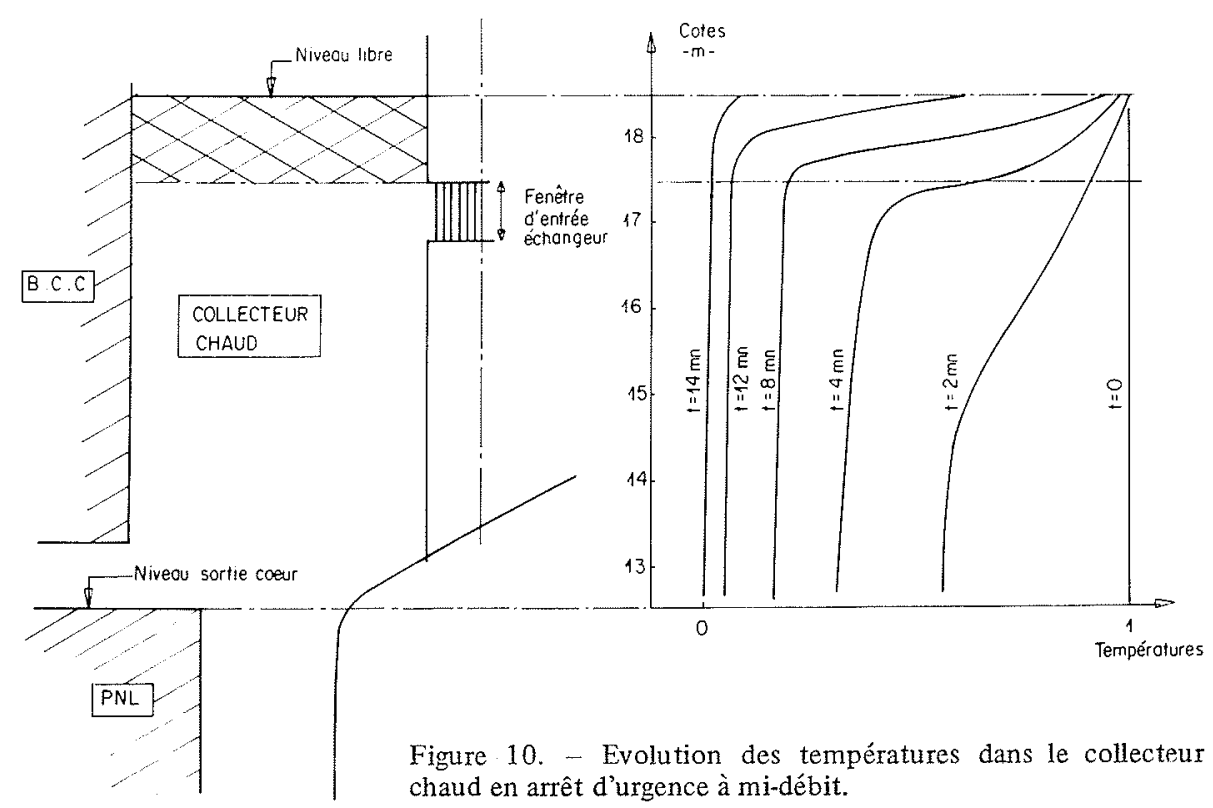

chaud en arrêt d'urgence à mi-débit. 


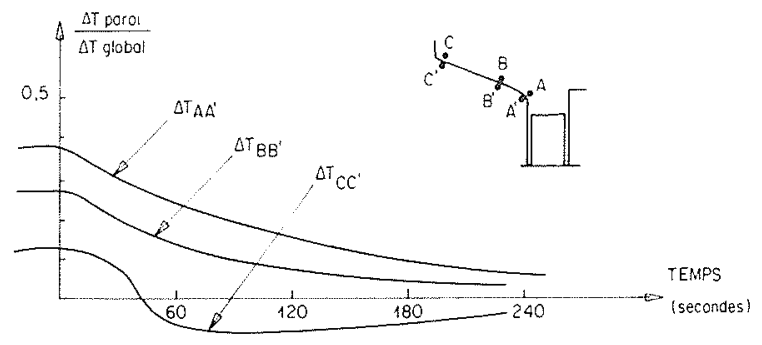

Figure 11 - Evolution de l'écart de température entre la peau chaude et la peau froide du redan pendant le transitoire.

les profils de température à différents instants. La zone chaude s'érode lentement sous l'effet de l'agitation turbulente créée par la circulation générale dans le collecteur chaud. Quant aux écarts de température à travers le redan, la figure 11 en montre l'évolution. C'est la variation entre l'écart maximal au cours du transitoire et l'écart au régime permanent qui est déterminant dans les calculs de structure.

\section{Champ thermique dans Super Phénix pendant un fonc- tionnement en "convection naturelle".}

\section{PRESENTATION DU PROBLEME}

A la suite d'un incident au cours duquel, successivement, les échangeurs intermédiaires sont hors service (les circuits secondaires sont vides de sodium), la puissance nominale dissipée dans le ccur réduite à la puissance résiduelle et les pompes primaires arrêtées, le refroidissement du cœur doit se faire par le seul moyen de la convection naturelle.

La charge motrice du thermosiphon dépend de la distribution de température dans le bloc réacteur, donc de la géométrie de celui-ci et du débit de sodium sortant du cour, et enfin du mode de refroidissement de la cuve extérieure.

Bien entendu, le débit et la température sont fonction à chaque instant aussi bien de ce qui se passe dans le cœur que dans le bloc réacteur.

\section{ETUDE ITERATIVE MODELE PHYSIQUE-CALCUL}

Le bloc réacteur est modélisé, en eau chaude, à l'échelle $1 / 20$. Le refroidissement extérieur par rayon- nement est simulé globalement par des échanges en convection naturelle dans l'air ambiant. Le modèle fournit l'évolution temporelle (découpage en zones) des températures dans le bloc réacteur, le débit et la température transitoires étant imposés à la sortie du cœur.

Ces résultats sont exploités par un code de calcul où l'on introduit le découpage par zone, en vue de recalculer la température et le débit à la sortie du cœur. Le processus itératif se poursuit par un nouvel essai sur le modèle.

Avec l'échelle géométrique retenue, l'échelle des temps, $\hat{\tau}$, est voisine de 1 . Au bout de 30 à 40 heures suivant les cas, la température de l'eau du modèle se stabilise, la puissance fournie équilibrant les pertes thermiques. L'essai se termine après un palier de quelques heures.

On présente sur la figure 12 un résultat d'essais. Pour le bloc réacteur considéré, on peut constater que le "découpage thermique" est simple : on distingue deux zones dont la frontière s'établit au niveau de retournement le plus bas du fluide chaud qui circule dans le modèle. Les visualisations et l'estimation des vitesses au voisinage de cette frontière montrent que la partie inférieure de la maquette ne s'échauffe que par conduction. A la partie supérieure, le fluide est faiblement stratifié avec un gradient vertical de $2^{\circ} \mathrm{C} / \mathrm{m}$ environ.

\section{Perspectives}

Nous avons présenté, à travers les cas précédents, les moyens dont on dispose actuellement pour traiter les problèmes de thermohydraulique présents dans les réacteurs surrégénérateurs refroidis au sodium.

Pour améliorer les connaissances, des études à caractère fondamental sont nécessaires. Il est en effet important de préciser la physique de certains phénomènes et notamment des écoulements stratifiés, compte tenu des particularités des transferts de chaleur en métaux liquides.

Pour appuyer ces réflexions, il est nécessaire de disposer d'études expérimentales en sodium représentatives des phénomènes et des paramètres adimensionnels rencontrés dans le réacteur. L'étude de l'interaction d'un

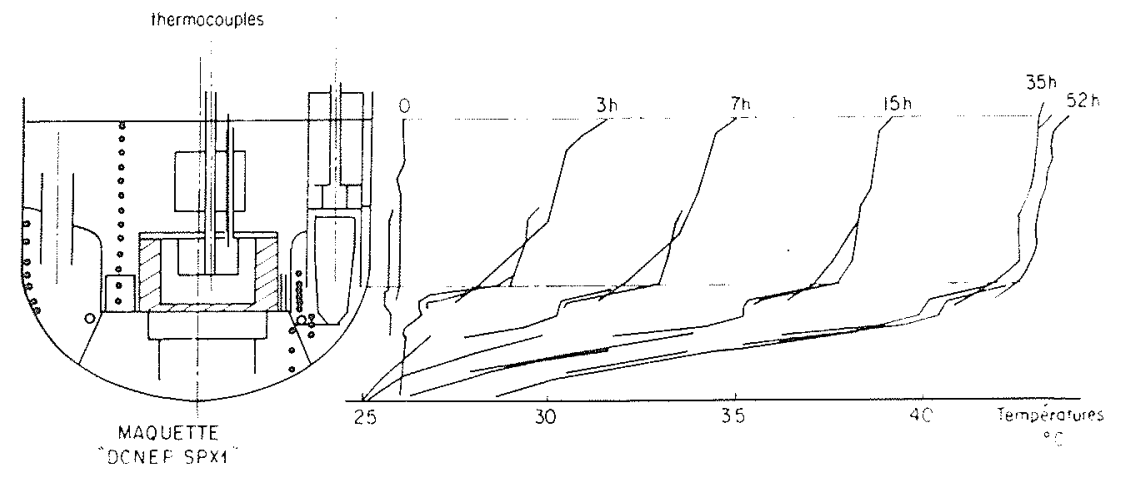

Figure 12 - Evolution des températures au cours d'un fonctionnement en convection națurelle. 
courant de recirculation avec un régime stratifié dans une cavité parallélépipédique a été engagée dans ce but.

Ces études à caractère fondamental doivent permettre d'améliorer les moyens dont on dispose actuellement: - les modèles physiques, en précisant mieux les distorsions inhérentes aux modèles réduits,

- les modèles numériques, en améliorant la simulation hydrodynamique et thermique de la turbulence.

\section{Références}

GRAND D. et VERNIER Ph. - 1) Forced convection heat transfer in liquid metals, 2) Combined convection in liquid metals, Turbulent forced convection in channels and rod bundles (Istambul, 20 juillet-30 août 1978 - NATO Advanced Study Institu te, Kakac S. Editors - Hemisphere).

LE FEVRE F.J., 1976. - Analysis of heat transfer from a vertical plane surface by turbulent natural convection, I.J. Heat Transfer - vol. 19, p. 1215-1216.

\section{Discussion}

\section{Président : M. M. BANAL}

Le Président remercie les conférenciers et ouvre la discussion.

M. HUETZ. - Cet exposé a été très riche et très intéressant. Dans le cas des mouvements déconnectés du champ de température en convection forcée turbulente, comment passez-vous du champ mécanique calculé au camp thermique ? Il faut avoir connaissance de la diffusivité turbulente des quantités de mouvements, de la diffusivité turbulente pour la chaleur et du Reynolds.

M. TAILLIFET. - Dans ces écoulements forcés, les flux de chaleur en dehors des couches limites sont essentiellement des transports convectifs, la diffusivité étant alors négligeable. Il est donc important de bien prendre en compte ces convections, donc de bien représenter le champ de vitesses. Dans le cas de l'échangeur, le champ thermique est régi par ces termes convectifs et les échanges représentés à l'aide des coefficients correspondants. Superposer à cela des termes de diffusivité ne modifierait pas le résultat.

M. GRAND. - A propos de la modélisation de la turbulence et de la diffusivité thermique qui en résulte, les métaux liquides, avec leur faible nombre de Prandtl, posent des problèmes spécifiques de simulation. Des modèles ont été proposés par différents auteurs dont vousmême. On peut donc introduire une diffusivité turbulente qui est une première approximation. Il faut également noter que les transferts turbulents de la chaleur dominant moins facilement dans un métal liquide que dans un fluide à nombre de Prandtl plus élevé, leur modélisation précise y est moins cruciale.

M. HUETZ. - Ma deuxième observation concerne une remarque que j'avais faite relativement aux épaisseurs de couches limites thermiques et mécaniques dans la convection naturelle le long d'une plaque plane chauffée, verticale.

Je suis d'accord sur le fait que, dans le cas du métal liquide, avec un Prandtl très faible, les épaisseurs de couches limites en convection forcée, relativement à la couche thermique d'une part, à la couche mécanique d'autre part, sont très différentes en première approximation; l'épaisseur de couche thermique rapportée à la couche limite mécanique est de l'ordre de Prandtl à la puissance $-1 / 3$.

Dans le cas de la convection naturelle, j'ai l'impression - et je voudrais avoir votre opinion à ce sujet - que les épaisseurs de couches limites doivent se rapprocher et même être égales quel que soit le type de fluide, et ceci selon le raisonnement suivant que je me permets d'exposer.

Soit une couche limite mécanique ; par définition, tout ce qui est dedans a une certaine vitesse. Mais si les couches thermiques s'étendent bien au-delà des couches mécaniques et si l'on suppose que $\theta_{\infty}$ est constant en milieu infini, on peut se faire le raisonnement suivant : tout ce qui se trouve dans la partie de la couche limite thermique supposée hors de la couche limite mécanique, subit une force ascensionnelle dirigée vers le haut et

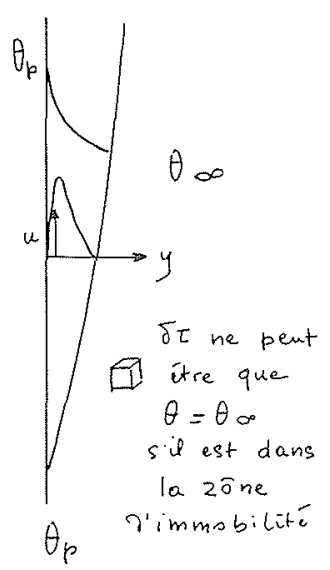

se déplace, donc se trouve dans la couche limite mécanique contrairement à l'hypothèse (schéma). La seule possibilité qui reste est que les couches limites mécanique et thermique soient égales.

M. GRAND. - Je suis d'accord avec votre remarque suivant laquelle il y aura écoulement en convection naturelle sur toute l'épaisseur de la couche limite thermique. J'ai voulu dire que les effets visqueux, eux, n'ont une influence que dans une région plus limitée au voisinage de la paroi.

M. HUETZ. - Est-ce que vous avez représenté le champ de vitesses?

M. GRAND. - La figure (ci-jointe) montre les profils de vitesse pour le régime laminaire et pour différents nombres de

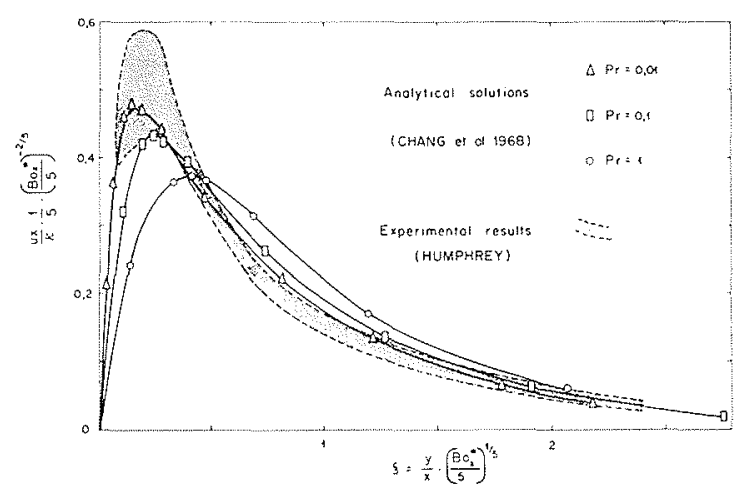


Prand tl. On distingue deux régions :

- A droite du maximum de vitesse (partie externe de la couche limite) les effets visqueux sont négligeables et le profil des vitesses résulte d'un équilibre entre les effets d'inertie et de flottabilité.

- Dans la partie comprise entre la paroi et le maximum de vitesse (partie interne) c'est l'équilibre entre les effets visqueux et de flottabilité qui conditionne le profil de vitesses.

M. HUETZ. - Le maximum de vitesse, en général, dans les couches limites mécaniques, est au tiers de l'épaisseur

M. GRAND. - Dans un métal liquide, il se rapproche de la paroi. Dans la figure où la coordonnée normale à la paroi est rapportée à l'épaisseur de la couche limite de convection naturelle, on voit que le maximum de vitesse situé au $1 / 3$ de l'épaisseur pour un nombre de Prandtl égal à 1 , est au $1 / 6$ de l'épaisseur environ quand $\operatorname{Pr}$ est égal à 0,01 . Le maximum de vitesse se rapproche asymptotiquement de la paroi quand le nombre de Prandtl tend vers zéro.

Le Président. - Vous avez parlé de DCNEP, c'est-à-dire Disparition des Circuits Normaux d'Evacuation de la Puissance. Il faut savoir que cela veut dire non seulement disparition de l'écoulement, mais également disparition du fluide.

M. GRAND. - Il en est ainsi dans les circuits secondaires, ce qui fait que les échangeurs intermédiaires cessent totalement leur effet.

Le Président. - Alors que, si le fluide subsistait, il continuerait à avoir, même en cas d'arrêt de pompes, un effet par convection naturelle. Il s'agit donc d'une hypothèse extrêmement sévère qui exige la rupture des quatre circuits secondaires. C'est un détail que je donne pour préciser un point qui avait été évoqué.

La Séance est levée à 17 h. 45 . 